\section{MR Imaging Findings of Infiltrating Lobular Carcinoma of the Breast}

\section{Abstract}

Purpose: Invasive lobular carcinoma (ILC) is the second most common malign breast neoplasia. Its classic histological type presents a slow and insidious growth, but no exuberant desmoplastic reaction. Therefore, its clinical and radiological detection is challenging. All the predictive characteristics for malignancy in breast cancer on magnetic resonance (MR) imaging are well established in the literature. However, there are few studies examining the peculiar image aspects of less prevalent histological subgroups such as the pure ILC case. Therefore, this paper assesses the characteristics of MR imaging results from patients with a histologically confirmed diagnosis of pure ILC by comparing them to the characteristics classically described in the literature for breast cancer in general.

Methodology: This document is an transversal study done on 43 female patients diagnosed with pure ILC who were treated at the Sírio-Libanês Hospital Mastology Center from January 2006 to August 2014 and who were subjected to pre-surgery and pre-biopsy MR examinations.

Results: Lesions presented an average size of $3.2 \mathrm{~cm}(0,6-10,0 \mathrm{~cm})$. The most common image aspect found was nodules with expansive growth pattern and irregular or spiculated margins. About $91 \%$ of patients showed nodules, and $9 \%$ showed only a non-mass enhancement (NME). Of patients with NME, the vast majority $(77,7 \%)$ showed segmental distribution and $22.3 \%$ were the focal type. Enhancement kinetics with type I curves were found on $30.3 \%$ of lesions. The most prevalent curve type was type II (51.1\%), while type III curves were only observed on $18.6 \%$ of lesions. In T2-weighted images, nearly half of the cases $(41 \%)$ showed a distinct low-intensity signal on $\mathrm{T} 2$, while the rest showed iso-intense signal. No lesions showed a high signal on T2-weighted images. Other data, such as the presence of macrovascularization and signs of edema and distortion surrounding the tumor were less prevalent and were observed in less than a third of patients.

Conclusion: In general, the image characteristics of pure ILCs imitate characteristics classically described for invasive breast cancer; however, a significant portion of ILC cases share less customary image characteristics including the presentation of nonmass enhancement and/or progressive enhancement, a distinct hypointense signal on T2-weighted images, and absence of macrovascularization or edema surrounding the tumor. Knowing these less customary aspects is an extremely important taskespecially in cases where abnormalities are evidenced exclusively through MRI.

Keywords: Breast Cancer, Invasive lobular carcinoma, Magnetic Resonance

Received: January 07, 2016; Accepted: February 12, 2016; Published: February 16, 2016
Marcos Fernando de Lima

Docema ${ }^{1}$,

Danúbia Ariana de Andrade², Adolfo Previdelli Bolinelli ${ }^{3}$, Valter Ribeiro dos Santos Junior ${ }^{3}$, Juliana Juliano Blandy Brockes ${ }^{4}$, Juliana Zabukas de Andrade ${ }^{2}$, Felipe Eduardo Martins de Andrade², Giovanni Guido Cerri $^{5}$ and Alfredo Carlos S. D. Barros ${ }^{6}$

1 Radiologist, Magnetic Resonance Department, Hospital Sírio Libanês, São Paulo, SP, Brazil

2 Mastologist, Mastology Department, Hospital Sírio Libanês, São Paulo, Brazil

3 Radiology Resident, Magnetic Resonance, Hospital Sírio Libanês, São Paulo, SP, Brazil

4 Radiologic Technologist, Magnetic Resonance, Hospital Sírio Libanês, São Paulo, SP, Brazil

5 Coordinator of the Imaging Diagnostics Department, Hospital Sírio Libanês, São Paulo, SP, Brazil

6 Coordinator of the Mastology Department, Hospital Sírio Libanês, São Paulo, Brazil

Corresponding author:

Marcos Fernando de Lima Docema

”mdocema@gmail.com

Radiologist, Magnetic Resonance Department, Hospital Sírio Libanês, São Paulo, SP, Brazil.

Tel: 551152125061

Citation: de Lima Docema MF, de Andrade DA, Bolinelli AP, et al. MR Imaging Findings of Infiltrating Lobular Carcinoma of the Breast. Ann Clin Lab Res. 2016, 4:1. 


\section{Introduction}

Invasive lobular carcinoma (ILC) is the second most common histological type among invasive neoplasm of the breast often ranging from 5 to $15 \%$ when considering their morphological variants $[1,2]$.

The macroscopic evaluation of the ILC may reveal a hard lesion with poorly defined and irregular contours, however, in some cases, the neoplasm is not palpable-even with diffuse involvement of the mammary tissue [2]. Microscopically, these tumors show a classic shape as well as variants due to cytological differences and stromal infiltration patterns by tumor cells. In its most typical form, the ILC is characterized by small tumor cells, relative uniformity, and isolated growth in a linear form ("single line") concentrically around the breast lobules and ducts ("target" growth formation) (Figures 1 and 2). The infiltration of the stroma may cause little or no desmoplastic reaction making it difficult to evaluate some lesions on physical examination, mammography and macroscopic studies [1-3]. One possible explanation for these features is the

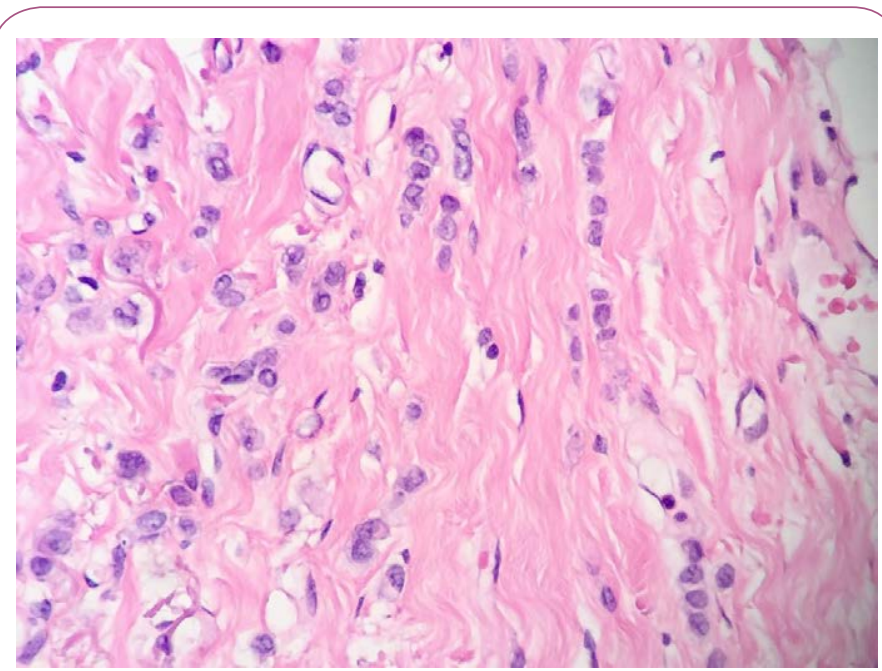

Figure 1 Invasive lobular carcinoma - infiltration in a "single line".

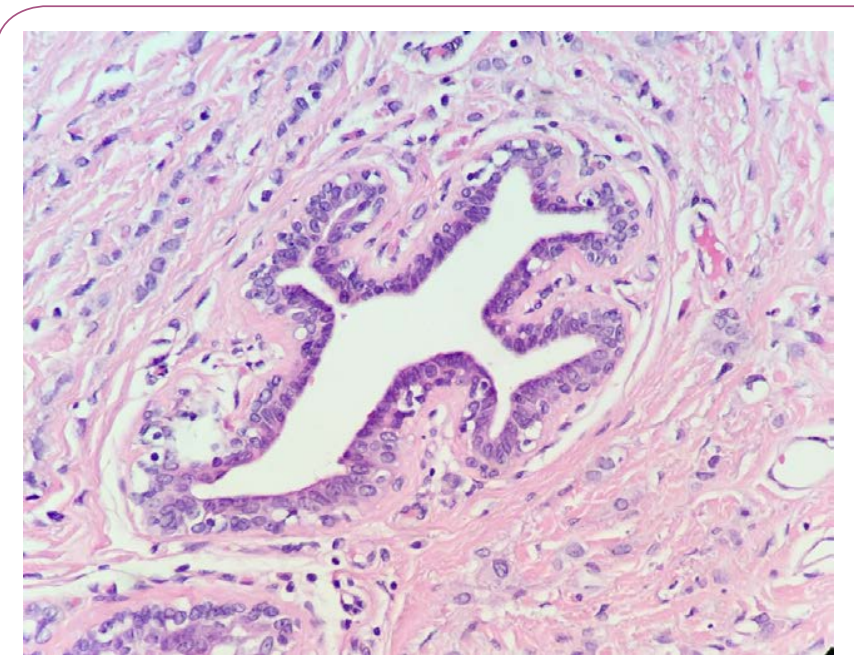

Figure 2

Invasive lobular carcinoma - arrangement of neoplastic cells "on target". loss of expression of the adhesion molecule E-cadherin due to mutations on the $\mathrm{CDH} 1$ gene, which leads to low cohesiveness between the tumor cells $[1,3]$.

In addition to this classic form, the ILC can be categorized according to the structural features in histological subtypes: trabecular, alveolar, solid variant, tubule-lobular and pleomorphic variants as well as additional subtypes based on cytological features (signet ring cells, cells with apocrine differentiation and histiocytic variants) [2] according to the histological grading of the Nottingham rating system $[4,5]$. The immunohistochemistry of the ILC are mostly positive for estrogen and progesterone receptors and are negative for HER-2. The expression of $p 53$ is unusual, cell proliferation rates are lower than those observed in ductal carcinomas $[2,4,5]$.

The ILC presents difficulties in clinical and radiological diagnosis mainly because of their pattern of infiltrative and insidious growth. Although the incidence of axillary involvement and ILC prognosis are similar to invasive carcinoma not otherwise specified (CAI SOE), there are differences in clinical presentation. In fact, $50 \%$ of patients with ILC have palpable abnormalities described as thickened and ill-defined areas rather than the typical nodule [6-12].

Mammography has sensitivity between $34 \%$ and $92 \%$ and tends to underestimate the size of the lesion. It has a craniocaudal incidence because the mammary parenchyma is most effectively compressed. Approximately $40 \%$ of speckled nodules displayed on the craniocaudal incidence appear as architectural distortions or asymmetries on the oblique mediolateral incidence.

While the most common presentation of ILC is the speckled or illdefined nodule (Figure 3), when compared to the CAI SOE, there is higher proportion of asymmetry or architectural distortion

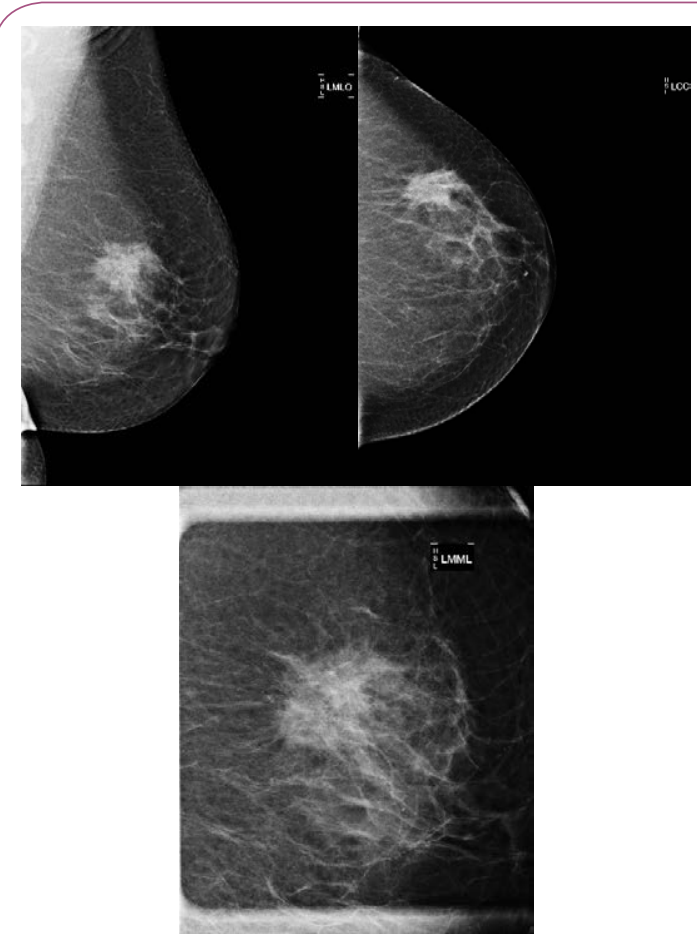

Figure 3 Irregular and spiculated nodule on the upper-outer quadrant of the left breast diagnosed with ILC in one of the patients. 
(Figure 4) rarely showing suspicious calcifications. An interesting feature of ILC is the possibility of decreasing the volume of the breast affected by the neoplasm during mammography. This is probably because of reduction in the natural elasticity of the tissue, which prevents it from spreading during compression [10-12].

Ultrasound has almost $92 \%$ sensitivity in detecting invasive lobular carcinoma depending on the array, which is much higher than mammography. Ultrasound finds hypoechoic nodules with irregular and ill-defined or speckled margins and rear acoustic shadowing (Figure 5) [11].

Magnetic resonance imaging (MRI) offers the highest sensitivity in detecting and detailing ILC [9]. The preoperative detection sensitivity is $\sim 93 \%$, but is less effective in retrospective studies due to its multifocality, multicentricity and bilaterality (Figures 6 and 7) $[9,11,13,14]$.

The American College of Radiology (ACR) provides a standardized vocabulary (BI-RADS ${ }^{\circledR}$ ATLAS BREAST MRI) for studying breast by MRI. This seeks to create greater uniformity among radiologists and collates the main predictive findings of malignancy. However, the aspects of unique images and less prevalent histological subgroups are still relatively poorly studied including pure ILC [15].

The objective of this study was to retrospectively analyze consecutive cases of patients with surgical confirmation of pure invasive lobular breast carcinoma on patients treated at the Philanthropy Clinic of Hospital Sírio-Libanês who underwent preoperative MRI to describe and identify the imaginological characteristics with higher prevalence on this histological subtype (Figures 8-10).
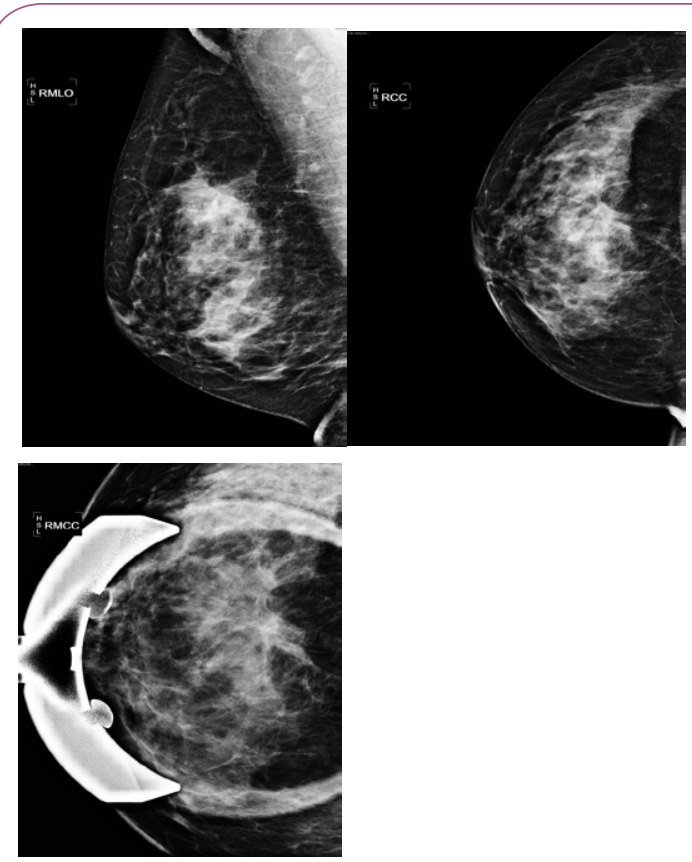

Figure 4 ILC characterized by architectural distortion in the middle third of the transition of the upper quadrants of the right breast.

\section{Methodology}

\section{Study design}

This is a cross-sectional study.

\section{Study population}

We conducted a sample including all patients diagnosed with pure breast ILC admitted at the Mastology Core of the Philanthropy Clinic at Sírio-Libanês Hospital between January 2006 and August 2014 and who underwent preoperative MRI.

\section{Inclusion criteria}

All patients with a diagnosis of invasive lobular breast carcinoma who underwent preoperative MRI before biopsy were included.

\section{Exclusion criteria}

All patients with different subtypes of breast carcinoma-other than the pure lobular carcinoma-with metastases at the diagnosis of cancer or primary neoadjuvant systemic therapy were excluded.

\section{Obtaining data}

Data came from electronic medical records of patients and image files on the PACS service (Picture Archiving and Communication System). All tests were issued by experts with at least 6 years of

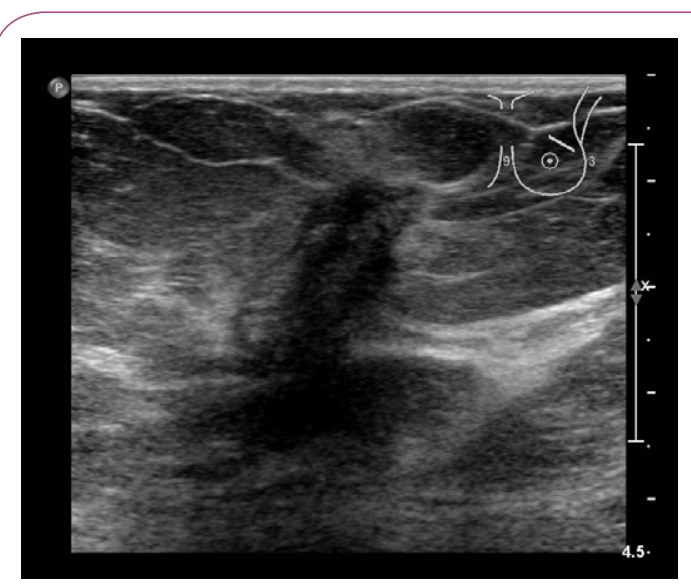

Figure 5 Irregular solid nodule on the upper-outer quadrant of the left breast in one of the patients diagnosed with ILC.

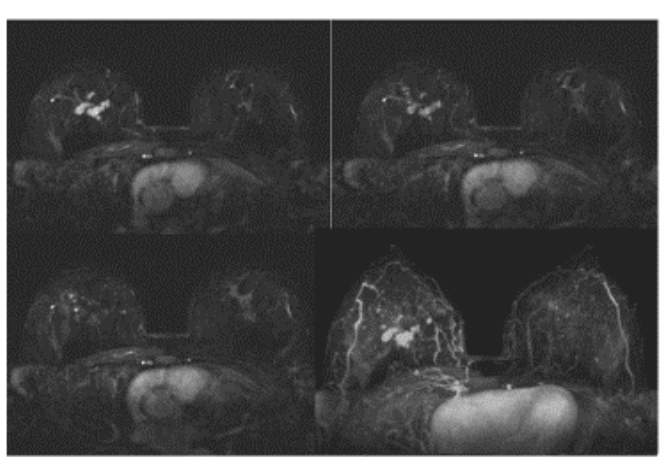

Figure 6

Multifocality: Multiple solid nodules with irregular contours and varying sizes on the right breast. 


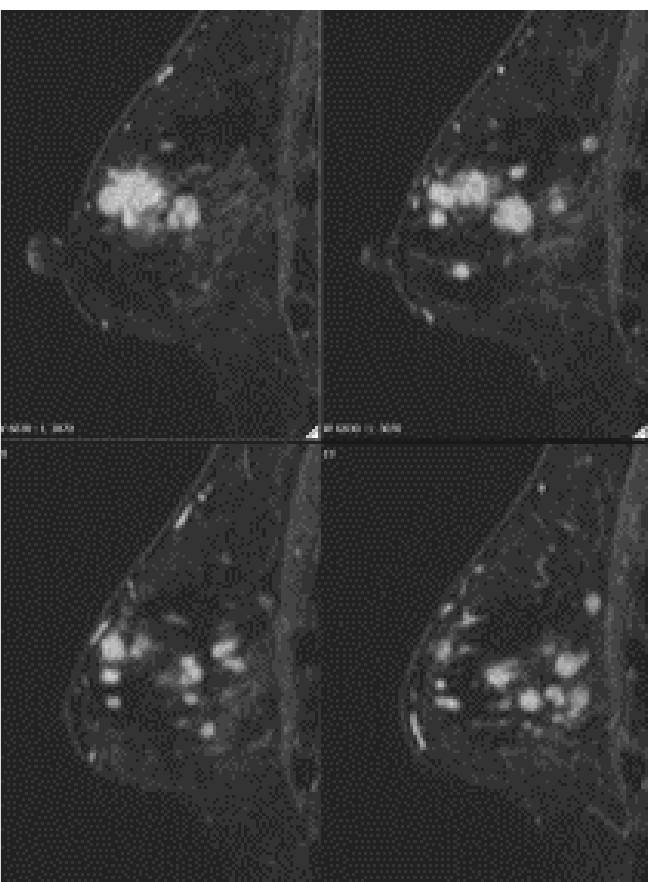

Figure 7 Multicentricity: Multiple solid nodules with irregular contours and various sizes in different breast quadrants. Reformatting the sagittal plane of the left breast.

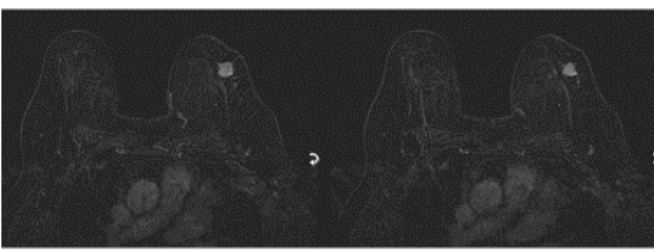

Figure 8 Patient with a unicentric nodule with expansive growth patterns and irregular contours.

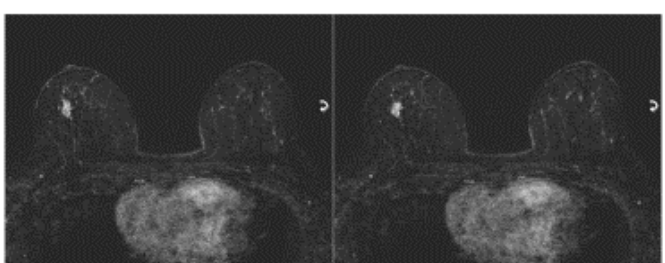

Figure 9 Patient of the study featuring a unicentric nodule with expansive growth pattern and spiculated contours.

experience and reviewed by a senior expert with nearly 14 years of experience in breast MRI analysis.

\section{Statistical analysis}

Quantitative variables were described using a central tendency (median, average), and the categorical variables were described through absolute and relative frequencies.

\section{Ethical aspects}

The data was collected from medical records by the researchers

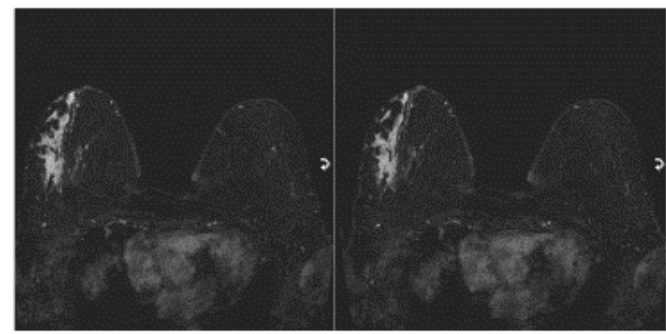

Figure 10 Non-nodular segmental distribution enhancement in the right breast; axial view.

themselves who were blinded to patient identification. The study was approved via the Consent and Information Form (TCLE) with the Research Ethics Committee of the Sírio-Libanês Hospital.

\section{Results}

During the study period, 1,401 patients were diagnosed with invasive breast carcinoma, and 86 patients (6.1\%) experienced pure ILC. Of these, 57 patients underwent breast MRI as a preoperative examination at Hospital Sírio-Libanês, and 14 were excluded because they held primary systemic therapy (neoadjuvant). Of the 43 patients studied, none were diagnosed with metastasis.

The median age at diagnosis was 59.3 years (range of $35-90$ years) and the average age was 59 years. Most patients (67.4\%) were diagnosed after menopause.

There were 42 patients with mammography data. Most patients (59.1\%) showed suspicious nodules and focal asymmetries, but in 4 cases $(7.4 \%)$ there were no change. In two cases, ultrasonography was normal (3.7\%).

The ultrasound data were described in 37 patients (Graph 2). In 30 cases $(81 \%)$, the echographic expression was inone nodule. There was architectural distortion in 3 cases (8.1\%) and 4 cases (10.8\%) had no alteration (Table 1).

In MRI, breast glandularity was characterized as fatty in $32.5 \%$ with scattered fibroglandular tissue in $37.2 \%$ with heterogeneous fibroglandular tissue in $25.5 \%$ and extreme fibroglandular tissue in $4.6 \%$ of cases. In most cases (65.1\%), there was parenchymal enhancement that was mild in $48.8 \%$ and late in $82.1 \%$ of cases. The average thickness of the breasts was $9.5 \mathrm{~cm}$, range of 3.4 to $15.9 \mathrm{~cm}$ (Table 2).

About MRI cancer features, the most common morphology was a nodule with an expansive growth pattern and irregular or spiculated margins. Approximately $91 \%$ of patients had a nodule or nodules association with non-mass enhancement; $9 \%$ (10 patients) had only non-mass enhancement (NME). Of NME patients, the large majority $(77.7 \%)$ had segmental distribution with $22.3 \%$ of those focal. The other forms of distribution known for non-mass enhancement were not found in this string of patients. There were 10 patients with standard non-mass enhancement-60\% had slow persistent enhancement kinetics. Expansive growth was observed in $72 \%$ and infiltrative in $18.6 \%$. Both were present in $9.3 \%$ of patients. Lesion size ranged from 0.6 to $10.0 \mathrm{~cm}$ with an average of $3.2 \mathrm{~cm}$. 
Table 1 Demographic and clinical features of ILC patients.

\begin{tabular}{|c|c|}
\hline Characteristics & Patients \% (n) \\
\hline \multicolumn{2}{|l|}{ Age } \\
\hline$<40$ years & $6.9(3)$ \\
\hline $40-49$ years & $11.6(5)$ \\
\hline $50-70$ years & $55.8(24)$ \\
\hline$>70$ years & $25.5(11)$ \\
\hline \multicolumn{2}{|l|}{ Menopausal Status } \\
\hline Pre menopause & $32.5(14)$ \\
\hline Post menopause & $67.4(29)$ \\
\hline \multicolumn{2}{|l|}{ Mammographic Findings } \\
\hline Nodule & $35.1(15)$ \\
\hline Assimetry & $24.0(10)$ \\
\hline Architectural Distortion & $20.3(8)$ \\
\hline Microcalcifications & $12.9(5)$ \\
\hline Normal & $7.4(4)$ \\
\hline \multicolumn{2}{|l|}{ Ultrasonography Findings } \\
\hline Nodule & $81.0(30)$ \\
\hline Architectural Distortion & $8.1(3)$ \\
\hline Normal & $10.8(4)$ \\
\hline
\end{tabular}

Table 2 MRI findings of ILC patients - General breast features.

\begin{tabular}{|c|c|}
\hline \multicolumn{1}{|c|}{$\begin{array}{c}\text { Characteristics } \\
\text { Mammary Glandularity }\end{array}$} & Patients \% (n) \\
\hline Almost entirely fat & $32.5(14)$ \\
\hline Scattered fibroglandular & $37.2(16)$ \\
\hline Heterogeneous fibroglandular & $25.5(11)$ \\
\hline Extreme fibroglandular & $4.6(2)$ \\
\hline $\begin{array}{c}\text { Background parenchymal enhancement (intensity) } \\
\text { Minimal }\end{array}$ & $34.8(15)$ \\
\hline Mild & $48.8(21)$ \\
\hline Moderate & $11.6(5)$ \\
\hline Marked & $4.6(2)$ \\
\hline Background parenchymal enhancement (kinetics) & $82.1(35)$ \\
\hline Late & $17.9(8)$ \\
\hline Early & $9.5 \mathrm{~cm} \mathrm{(3.4} \mathrm{-} \mathrm{15.9)}$ \\
\hline Thickness of the breasts & \\
\hline Average (range) &
\end{tabular}

The T2 signal intensity showed that $58 \%$ of patients had isointense signal cancer with normal surrounding parenchyma; $41 \%$ of patients had malignant findings with low-intensity signal on T2. No patients showed high T2 signal. Type I enhancement curve were found in $30.3 \%$ of the lesions. The most prevalent was the type II curve (51.1\%), and the type III curve was seen in $18.6 \%$ (Table 3).

We also noted characteristics of the parenchyma adjacent to the tumor as the degree of hydration, presence of architectural distortion and overall macrovascularization of the breasts (Table 4).

Onanatomopathologicalevaluation,40patients(93\%)experienced classic ILC and 3 patients experienced the pleomorphic variant ILC (6.9\%). Most tumors showed unifocality (65.1\%) associated with an in situ component (72\%), histological grade $2(76.7 \%)$, and nuclear grade 2 (74.4\%) with an absence of angiolymphatic invasion (83.7\%). The tumor size ranged from 1.3 to $86 \mathrm{~mm}$ with
Table 3 MRI findings of ILC patients - Cancer features.

\begin{tabular}{|c|c|}
\hline $\begin{array}{c}\text { Characteristics } \\
\text { Type of the lesions }\end{array}$ & Patients $\%(\mathbf{n})$ \\
\hline Nodule & $79.0(34)$ \\
\hline Non-mass enhancement & $9.3(4)$ \\
\hline Both & $11.6(5)$ \\
\hline Characteristics of the nodule margins & \\
\hline Irregular & $64.1(22)$ \\
\hline Spiculated & $35.8(12)$ \\
\hline T2 signal intensity & \\
\hline T2 hypointense & $41.9(18)$ \\
\hline T2 isointense & $58.1(25)$ \\
\hline T2 hyperintense & $0(0)$ \\
\hline Kinetics of enhancement & $30.0(13)$ \\
\hline Type I & $51.1(22)$ \\
\hline Type II & $18.6(8)$ \\
\hline Type III & $13,9(6)$ \\
\hline Tumor $s i z e$ & $27.9(12)$ \\
\hline$\leq 1.0 \mathrm{~cm}$ & $41.8(18)$ \\
\hline$>1.0$ to $2.0 \mathrm{~cm}$ & $16.2(7)$ \\
\hline$>2.0$ to $5.0 \mathrm{~cm}$ & \\
\hline$>5.0 \mathrm{~cm}$ & \\
\hline
\end{tabular}

an average of $31.1 \mathrm{~mm}$. Regarding immunohistochemistry, most tumors were positive for estrogen and progesterone receptors (79\%) and no absence HER-2 expression (90.6\%) and $67 \leq 14 \% \mathrm{KI}$ (48.8\%). No cases showed immuno-expression to the E-cadherin molecule.

\section{Discussion}

In this study, the percent of cases diagnosed as ILC was similar to previous studies (6.1\%). Clinical data such as age at diagnosis, menopausal status, tumor size, histological grade, angiolymphatic invasion and positive results for hormone receptors were similar to the literature $[4,14]$. The main goal of study was to describe and identify the most prevalent MRI features of pure ILC.

MRI is increasingly being used as a complement to mammography and ultrasound because it improves the detection and characterization of breast tumors-especially lobular carcinomas. The sensitivity of MRI for breast cancer detection is very highnearly $93 \%$ in most studies. However, false-negative results can occur in the presence of ILC making it an insidious disease with variations in morphology and kinetics. In this series, there was a sensitivity of $100 \%$; all examinations were done preoperatively $[13,15]$. In this study, two patients $(4.5 \%)$ had normal mammography and ultrasound screening and only MRI could diagnose the lesion.

After evaluating the signal intensity of the lesions on $\mathrm{T} 2,58 \%$ of patients had cancer with iso-intense signal to the normal surrounding parenchyma. While not a major criterion for predicting malignancy, this is the most commonly identified feature of invasive ductal carcinomas. Approximately $42 \%$ of the patients had malignant findings with low-intensity signal on T2. None showed high signal associated with the ILC, which is commonly related to benign findings of the breasts or seen in the 
Table 4 MRI findings of ILC patients - Adjacent parenchymal features.

\begin{tabular}{|c|c|}
\hline $\begin{array}{c}\text { Characteristics } \\
\text { Increase of parenchymal hydration }\end{array}$ & Patients $\%$ (n) \\
\hline No & $83.7(36)$ \\
\hline Yes & $16.2(7)$ \\
\hline Parenchymal distortion & $74.4(32)$ \\
\hline No & $25.5(11)$ \\
\hline Yes & \\
\hline Macrovascularization & $81.3(35)$ \\
\hline Habitual & $18.6(8)$ \\
\hline Increased
\end{tabular}

presence of tumors with special subtypes $[15,16]$.

Regarding the morphology, ILC is no different from other malignant histological subtypes with nodules being the most common feature. The main features were nodules with irregular borders (61.5\%) or spiculated (35.8\%); Mann et al., reported that the percentage of cases in which a nodule was found can reach $31 \%$ to $95 \%$ [11]. Caramella et al., analyzed the MRI findings of 57 patients with ILC and reported that the most common findings were irregular and spiculated nodules [16].

We emphasize that although the morphology of most common ILCs in our series of cases were the presence of nodules with expansive growth patterns and irregular or spiculated margins, about $9 \%$ were characterized by a non-mass enhancement. This pattern makes diagnosis difficult and prevents conventional characterization. Of the tumors characterized by non-mass enhancements, the large majority $(77.7 \%)$ showed segmental distribution, which is the MRI finding most commonly associated with DCIS. Thomasin-Naggara et al., showed that $80 \%$ of cases of non-mass enhancement with segmental distribution were confirmed as malignant results (high VPP for malignancy). Of these, $64.0 \%$ were DCIS [18]. In our case series, in 10 patients with non-mass enhancement findings, 4 had focal asymmetry in mammograms, 3 presented architectural distortion on mammogram or ultrasound, 2 presented nodules, and one patient presented clustered microcalcifications. Six of these also showed progressive kinetics enhancement, which further hindered diagnosis.

According to the literature, the kinetics enhancement curve of ILC to the dynamic study of type III is the most specific for malignancy but is not prevailing. In this series of cases, we found a type III curve in $18.6 \%$ of the patients. The type II curve was found in $51.1 \%$ of patients and type I in $30 \%$. According to Caramella et al., only $49 \%$ of ILC showed a standard kinetic curve that indicated malignancy Kim et al., [17] Reported a type II curve in $73 \%$ of cases and type III in 23.1 [16]. Here, nearly one third of patients showed the slow and persistent enhancement (type I curve) that is most commonly associated with benign findings and that can be explained by the slow and infiltrative tumor growth not inducing exuberant neovascularization. This is also seen in the low levels of vascular endothelial growth on tumors with lobular histology. This indicates a different signaling pathway in the neovascular formation that results in capillaries with less leakage and consequently slower enhancement [13].
This set of findings may also explain the low prevalence of global macrovascularization in the affected breast and the low incidence of edema on surrounding ILC parenchyma. We also observed that only $25 \%$ of patients presented associated architectural distortion.

There are also indications that these characteristics are peculiar to the lobular carcinoma. One possible explanation is low levels of a lack of desmoplastic reactions in the parenchyma caused by this tumor [15].

Another aspect is the assessment of the extent of disease by MRI with relatively high reliability. Here, the size of the lesions observed by MRI ranged from 0.6 to $10.0 \mathrm{~cm}$ (average of 3.2 $\mathrm{cm}$ ). These data are apparently consistent with pathological examination where the average tumor size was $3.1 \mathrm{~cm}$. Muttalib et al., reported that tumors were underestimated by MRI in $59.1 \%$ of cases and overestimated in $34.1 \%$ of cases [19]. Even though the understimation and overestimation of the lesion size by MRI still occurs, this method is the most accurate diagnostic and has a high detection rate of tumor size [11].

In this series, the lesions were multifocal in $11.8 \%$ of cases, multicentric in $13.9 \%$ of cases and multifocal/multicentric in $2.3 \%$ of the patients; tumors were bilateral in 6 patients (13.9\%). According to the literature, MRI has a high accuracy in the detection of synchronous lesions in the affected breast or contralateral breast ranging from 80 to $90 \%$. This facilitates a better surgical approach and is key to the indication and planning of possible neoadjuvant chemotherapy [20-23].

Despite evidence for the high sensitivity of MRI in the detection of ILC, its role in preoperative staging with conservative surgery is controversial and lacks randomized clinical trials to assess the impact on overall survival. An argument against the use of MRI as a preoperative tool is the claim that the additional disease diagnosed by MRI would respond to the adjuvant treatment (chemotherapy and/or radiotherapy) without the need for additional surgical procedures. However, most synchronous lesions diagnosed by MRI have unfavorable histological characteristics and prognoses similar to the index lesion.18 Randomized clinical trials show the benefit of MRI in reducing the rapprochement rates in conservative breast surgery without increasing the number of mastectomies in ILC [19,21,22,23].

\section{Conclusion}

We conducted a cross-sectional study to identify the imaging characteristics of pure ILC on MRI scans using a series of 43 consecutive cases with histologically-confirmed diagnosis. The data demonstrate that the aspects classically associated with breast cancer in MRI (nodules with irregular or spiculated margins) are also the most prevalent in the pure ILC subgroup. However, a considerable portion of the ILCs have less common imaging features including features typical of invasive ductal carcinoma. These features include enhancement with a non-mass pattern and/or kinetics of slow progressive enhancement. Coincidentally, we found that most of these lesions had focal asymmetry or architectural distortion to conventional methods, which further hinders its diagnosis prior to MRI. 
There was less macrovascularization and the edema around the tumor than previously reported for ductal carcinoma suggesting correlation with ILC characteristics. The lesions' T2 signal behavior, while not discriminating, showed many cases with low signal. No lesion had high T2 signal. The number of false-negative cases can be decreased by knowing the features that are less commonly described in connection to breast cancer in general, but that may be present in cases of invasive tumors of the lobular subtype. This is especially important in cases where the abnormalities are highlighted exclusively by MRI. Complementary comparative studies are necessary including subgroups with ductal and ductal in situ carcinomas. 


\section{References}

1 Harris JR, Lippman ME, Morrow M, Osborne CK (2014) Disease of the breast. (5th edn) Philadelphia: Wolters Kluwer Health, USA.

2 Dabbs DJ (2012) Breast Pathology (1stedn). Pittsburgh: Elsevier, USA.

3 Rosai J (2011) Ackerman's Surgical Pathology (10thedn). Philadelphia: Elsevier, USA.

4 Orvieto E, Maiorano E, Bottiglieri L, Maisonneuve P, Rotmensz N, et al. (2008) Clinic pathologic characteristics of invasive lobular carcinoma of the breast: Results of an analysis of 530 cases from a single institution. Cancer 113: 1511-1520.

5 Rakha EA, El-Sayed ME, Menon S, Green AR, Lee AH, et al. (2008) Histologic grading is an independent prognostic factor in invasive lobular carcinoma of the breast. Breast Cancer Res Treat 111: 121127.

6 Schnitt SJ, Shin SJ (2012) Breast Pathology: Diagnostic Dilemmas. Surg Pathol Clin 5: ix.

7 Bassett LW, Mahoney MC, Apple S, Orsi C (2011) Breast imaging (2ndedn). Philadelphia: Elsevier, USA.

8 Bland KI, Copeland EM (2009) The breast (4thedn) Philadelphia: Elsevier, USA.

9 Winchester DJ, Chang HR, Graves TA, Menck HR, Bland KI, et al. (1998) A comparative analysis of lobular and ductal carcinoma of the breast: presentation, treatment, and outcomes. J Am Coll Surg 186: 416-422.

10 Molleran VM, Mahoney MC (2014) Breast MRI (1stedn). Philadelphia: Saunders Elsevier, USA.

11 Mann RM, Hoogeveen YL, Blickman JG, Boetes C (2008) MRI compared to conventional diagnostic work-up in the detection and evaluation of invasive lobular carcinoma of the breast: a review of existing literature. Breast Cancer Res Treat 107: 1-14.

12 (2013) The American College of Radiology. BI-RADS ${ }^{\circledR}$ ATLAS (5thedn). USA.

13 Yeh ED, Slanetz PJ, Edmister WB, Talele A, Monticciolo D, et al. (2003) Invasive lobular carcinoma: spectrum of enhancement and morphology on magnetic resonance imaging. Breast J 9: 13-18.
14 Pestalozzi BC (2008) International Breast Cancer Study Group. Distinct clinical and prognostic features of infiltrating lobular carcinoma of the breast: Combined results of 15 international breast cancer study group clinical trials. J Clin Oncol 26: 3006-3014.

15 Macura KJ, Ouwerkerk R, Jacobs MA, Bluemke DA (2006) Patterns of enhancement on breast MR images: interpretation and imaging pitfalls. Radiographics 26: 1719-1734.

16 Caramella T, Chapellier C, Ettore F, Raoust I, Chamorey E, et al. (2007) Value of MRI in the surgical planning of invasive lobular breast carcinoma: a prospective and a retrospective study of 57 casescomparison with physical examination, conventional imaging, and histology. Clin Imaging 31: 155-161.

17 Kim SH, Cha ES, Park CS, Kang BJ, Whang IY, et al. (2011) Imaging features of invasive lobular carcinoma: comparison with invasive ductal carcinoma. Jpn J Radiol 29: 475-482.

18 Thomassin-Naggara I, Trop I, Chopier J, David J, Lalonde L, et al. (2011) Nonmasslike enhancement at breast MR imaging: the added value of mammography and US for lesion categorization. Radiology 261: 69-79.

19 Muttalib M, Ibrahem R, Khashan AS, Hajaj M (2014) Prospective MRI assessment for invasive lobular breast cancer. Correlation with tumour size at histopathology and influence on surgical management. Clin Radiol 69: 23-28.

20 Oliveira TM, Elias JJ, Melo AF, Teixeira SR, Filho SC, et al. (2014) Evolving concepts in breast lobular neoplasia and invasive lobular carcinoma and their impact in imaging methods. Insights Imaging 5: 183-194.

21 Mann RM, Loo CE, Wobbes T, Bult P, Barentsz JO, et al. (2010) The impact of preoperative breast MRI on the re-excision rate in invasive lobular carcinoma of the breast. Breast Cancer Res Treat 119: 415422.

22 Kneeshaw PJ, Turnbull LW, Smith A, Drew PJ (2003) Dynamic contrast enhanced magnetic resonance imaging aids the surgical management of invasive lobular breast cancer. Eur J Surg Oncol 29: 32-37.

23 Boetes C, Veltman J, van Die L, Bult P, Wobbes T, et al. (2004) The role of MRI in invasive lobular carcinoma. Breast Cancer Res Treat 86: 31-37. 